\title{
Prevalence of onchocerciasis and associated clinical manifestations in selected hypoendemic communities in Ghana following long-term administration of ivermectin
}

Kenneth Bentum Otabil ${ }^{1,2^{*}}$ (D), Samuel Fosu Gyasi ${ }^{1}$, Esi Awuah ${ }^{3}$, Daniels Obeng-Ofori ${ }^{4}$, Robert Junior Atta-Nyarko ${ }^{5}$, Dominic Andoh ${ }^{5}$, Beatrice Conduah ${ }^{5}$, Lawrence Agbenyikey ${ }^{5}$, Philip Aseidu ${ }^{5}$, Comfort Blessing Ankrah,

Abdul Razak Nuhu ${ }^{5}$ and H. D. F. H. Schallig ${ }^{2}$

\begin{abstract}
Background: Onchocerciasis is a neglected tropical disease which is still of immense major public health concern in several areas of Africa and the Americas. The disease manifests either as ocular or as dermal onchocerciasis with several symptoms including itching, nodules, skin thickening, visual impairment and blindness. Ivermectin has been an efficient microfilaricide against the causative agent of the disease (Onchocerca volvulus) but reports from some areas in Africa suggest the development of resistance to this drug. The aim of this study was to determine the prevalence of onchocerciasis and associated clinical conditions frequently associated with the disease in three endemic communities in Ghana which have been subjected to 18 to 20 rounds of mass drug administration of ivermectin. This was to help determine whether or not onchocerciasis persists in these communities.

Methods: A cross-sectional study design was adopted. Three communities (Tanfiano, Senya and Kokompe) in the Nkoranza North District of Ghana where mass drug administration of ivermectin had been ongoing for more than two decades were selected for the study. The population was randomly sampled and 114 participants recruited for the study based on the eligibility criteria. The study participants were examined for the presence of parasites and clinical manifestations of onchocerciasis following established protocols.
\end{abstract}

Results: The study showed that the prevalence of microfilaria in the Tanfiano, Senya, Kokompe communities were 13.2, 2.4, and 2.9\%, with nodule prevalence being 5.3, 4.9 and $14.3 \%$ respectively. Females in the study communities had a higher prevalence of microfilaria carriers (5.17\%) relative to males (2.44\%), but this difference was not statistically significant $(p=0.2800$, unpaired $t$ test). The most frequent clinical manifestation observed in this study among all participants was dermatitis (25.4\%), followed by visual impairment \& nodules (7.9\% each) and then by blindness (4.4\%).

(Continued on next page)

\footnotetext{
*Correspondence: k.b.otabil@amc.uva.nl

${ }^{1}$ Department of Basic and Applied Biology, School of Science, University of

Energy and Natural Resources, Sunyani, Ghana

${ }^{2}$ Amsterdam University Medical Centres, Academic Medical Centre, University

of Amsterdam, Department of Medical Microbiology, Experimental

Parasitology Unit, Amsterdam, The Netherlands

Full list of author information is available at the end of the article
}

(c) The Author(s). 2019 Open Access This article is distributed under the terms of the Creative Commons Attribution 4.0 International License (http://creativecommons.org/licenses/by/4.0/), which permits unrestricted use, distribution, and reproduction in any medium, provided you give appropriate credit to the original author(s) and the source, provide a link to the Creative Commons license, and indicate if changes were made. The Creative Commons Public Domain Dedication waiver (http://creativecommons.org/publicdomain/zero/1.0/) applies to the data made available in this article, unless otherwise stated. 
(Continued from previous page)

Conclusion: The study showed that despite several years of mass drug administration with ivermectin, infection with onchocerciasis and the commonly associated clinical manifestations of the disease still persist in the study communities. This calls for a greater urgency for research and development aimed at discovering new or repurposed anti-filarial agents which will augment ivermectin if global onchocerciasis eradication targets are to be achieved.

Keywords: Onchocerciasis, Nodules, Dermatitis, Hypoendemic areas, Neglected tropical disease, Ocular onchocerciasis

\section{Background}

Onchocerciasis is the world's second largest cause of infectious blindness and a major public health problem in many parts of the world [1]. The disease is caused by Onchocerca volvulus parasites and endemic in 37 countries in West, East and Central Africa, the Arabian Peninsula and parts of South and Central America [1]. Worldwide, about 90 million people are at risk of contracting the disease in endemic areas mainly in sub Saharan Africa, of which more than 37 million are estimated to be infected and 300,000 are permanently blind as a consequence of onchocerciasis [1] . However, these figures are generally accepted to be an underestimation of the true situation [2].

Although rarely life threatening, onchocerciasis causes chronic suffering and severe disability with approximately 1.5 million disability-adjusted life-years (DALYs) being lost each year due to this disease [3] . Severe pruritis associated with the disease alone accounts for $60 \%$ of these DALYs [3]. In some hyperendemic communities, every second person infected ultimately goes blind [4]. Moreover, infection with Onchocerca worms reduces the host's immunity and resistance to other diseases, resulting in an estimated reduction in life expectancy of 13 years [3]. If many people are infected in a community, socioeconomic depression may result as fertile riverine areas are frequently abandoned for fear of the disease [1].

Clinical features of the disease include both skin and ocular involvement. Typically, skin involvement consists of intense itching, swelling, and inflammation [1]. Skin changes include acute papular onchodermatitis, chronic papular onchodermatitis, hyperpigmentation, lichenified onchodermatitis, lymphadenopathy, pruritus and common secondary bacterial infections; Skin atrophy; depigmentation, usually on anterior lower leg [5]. Ocular involvement provides the common name associated with onchocerciasis - river blindness and may involve any part of the eye from conjunctiva and cornea to uvea and posterior segment including retina and optic nerve [5].

Currently, the only drug available to treat onchocerciasis is ivermectin (Mectizan TM, Merck) [6]. Ivermectin is a safe and effective microfilaricide and has since 1987 been successfully used in mass drug administration programs (MDA) aimed at both reducing the burden of disease and controlling transmission [6]. However, persistent microfilaridermia (microfilariae in the skin) and transmission have been reported after 15-20 years of ivermectin treatment in some areas of Africa [7-9]. Awadzi et al. (2004) working in Ghana identified O. volvulus worms that exhibited an ivermectin response phenotype termed sub-optimal response (SOR) [7, 10]. These SOR parasites were characterized by the presence of live stretched microfilariae in the uteri of the adult worms 90 days after treatment, and were associated with repopulation of the skin with microfilariae earlier and more extensively than expected based on prior data [10, 11]. Nevertheless, ivermectin exposure was found not to be an explanatory factor $[10,11]$. Additional investigations have reported this phenotype in other areas in Ghana $[2,9,12]$ and in Cameroon $[13,14]$. Allele frequency change in a number of candidate ivermectin response genes (chosen for analysis based on specific hypotheses concerning mechanisms of resistance to the acute effects of ivermectin in O. volvulus) has also been demonstrated in these populations when sampled before and after several rounds of ivermectin treatment $[8,15-19]$, which is consistent with population genetic changes associated with drug selection pressure [20]. While genetic selection for SOR was not demonstrated, these studies suggest that these populations are being influenced at the genetic level by ivermectin treatment and if these candidate genes mediate the phenotypic changes in ivermectin response then SOR might have a genetic basis that may involve selection on several genes [20].

In response to the increasing concerns on diminishing effectiveness of ivermectin, there is a need to keep monitoring the efficacy of ivermectin in onchocerciasis endemic areas. The present study aimed to investigate the prevalence of onchocerciasis and clinical manifestations commonly associated with the disease in three communities in the middle belt of Ghana that are endemic for onchocerciasis and have been subjected to 18 to 20 rounds of MDA. The study aimed to provide information on the persistence (or otherwise) of the disease and its clinical manifestations in the study communities. 


\section{Methods}

\section{Study area}

This study was done in 3 communities (Tanfiano, Senya and Kokompe) in the Nkoranza North District of the Bono East Region of Ghana (West Africa). The three communities were selected because no effective monitoring of the effectiveness of the MDA programmes in the communities had previously been performed in them, although ivermectin MDA had been ongoing in these endemic communities for several years. The communities were chosen to represent the Western (Tanfiano), central (kokrompe) and Eastern (Senya) communities of the District $[21,22]$. The Nkoranza North lies within longitudes $1^{\circ} 10^{\prime \prime} \mathrm{W}$ and $1^{\circ} 55^{\prime \prime} \mathrm{W}$ and latitudes $7^{\circ} 20^{\prime \prime} \mathrm{N}$ and $7^{\circ} 55^{\prime \prime}$ $\mathrm{N}[21,22]$. The Tanfi and Tanko Rivers in the area are fast flowing and provide suitable breeding grounds for the black flies which are the vectors of onchocerciasis. The major rainy season is between April and June and the minor rainy season occurs between September and November [21]. The temperature in the District is generally high with an annual average temperature of $26.0^{\circ} \mathrm{C}$. Average maximum temperature is $30.9{ }^{\circ} \mathrm{C}$ and minimum of $21.2^{\circ} \mathrm{C}$. The hottest months are February, March and April [21].

\section{Study design}

A cross-sectional study with a random sampling approach was employed. The study was part of a larger study which started in September 2017 and ended in February 2019. The cross-sectional (prevalence) study was performed in September 2017. A few days prior to the recruitment, announcements were made via the community information centers or by a 'gong-gong' beater (a local means of giving information to community residents where the 'beater' moves around sounding a metallic instrument 'the gong gong' whilst intermittently shouting out the information). Community residents were informed to gather at chosen social centers. The study was explained in English and then in the local language 'Twi' during the meeting with the village elders and interested villagers at a chosen gathering point in the community. The participants were invited to ask questions and these were answered. During the surveys, persons identified as eligible to participate in the study were informed individually in English by the research team and then in 'Twi'. Informed, signed/thumb printed, or witnessed consent was obtained from all participants. The Informed Consent Forms were in English and were explained in local language to participants who did could not read or write in English.

\section{Recruitment of study patients}

A total of 114 eligible individuals from all 3 communities were randomly sampled and recruited for this study, resulting in an average sample size of 38 individuals from each community. This meets the average sample size (around 40 per village) for most prevalence studies on onchocerciasis as reported by the WHO [23]. Individuals were made to randomly queue and all individuals who were in the queue were given the chance of being included in the study once they met the inclusion criteria. The criteria for inclusion in the study was that a participant should be a male or female, aged 18-95 years with willingness to participate in the study, indicated by signing an informed consent form. The informed consent form along with study protocol was approved by the scientific and ethical review Boards of the Kintampo Health Research Centre (KHRC) in Ghana. The approval number for the study is KHRCIEC/2018-18. Before parasitological and physical examinations of eligible participants, basic demographic data, i.e. name, gender, age and number of rounds of ivermectin were collected using the case report forms.

\section{Palpation}

Individuals were palpated to check for the presence of nodules (onchocercomas) harbouring adult worms of $O$. volvulus. Nodules were 'suspected' onchocercomas (not confirmed by dissection or biopsy) defined as being firm, often flattened or bean-shaped, usually movable, non-tender and up to several centimeters in diameter. They were distant from usual locations of lymph nodes (neck, axillae, inguinal) as the palpation were done on bony prominences of the ribs, iliac crests, sacrum and upper leg [24].

\section{Visual acuity tests}

All study participants were examined by medical personnel for possible vision loss suspected to be associated with past or ongoing infection with onchocerciasis using a Snellen chart to determine whether the volunteers had 20/20 vision or if they showed signs of visual impairment [25]. Visual impairment was defined as presenting with visual acuity worse than 6/12 (mild), 6/18 (moderate) or 6/60 (severe) whereas blindness was defined as presenting visual acuity worse than $3 / 60$. All examinations were done without corrective glasses.

\section{Physical examinations for signs of dermal onchocerciasis}

The study participants were further examined for the signs of dermal onchocerciasis and the results of these assessments were recorded in the case reports forms [24]. Dermal onchocerciasis was defined as any of the common skin manifestations of onchocerciasis. An example is dermatitis which is the first manifestation and maybe isolated or associated with skin lesions [26]. It can be troublesome and sometimes irresistible though scratching may lead to filarial scabies which is often 
confused with scabies, especially in countries where suspicion of onchocerciasis does not arise [26].

\section{Skin snipping and microscopy}

Tiny pieces of blood-free skin snips were obtained from all registered study participants from each community to determine individual microfilarial counts in order to evaluate the prevalence and intensity of infection in each community. A piece of cotton wool soaked in methylated spirit was used to clean both sides of the iliac-crest of each person. Blood-free skin snips (approx. $2 \mathrm{mg}$.) were taken from each individual using a sterilized corneoscleral punch. The biopsies were then incubated in physiological saline for microscopic examination to detect microfilariae (mf) of O. volvulus [2].

Microfilariae were counted using 10X objective lens of a compound microscope. The geometric means of the microfilaria from the 2 skin biopsies from each patient were calculated and used as a measure of intensity of infection. The standard prevalence was also determined as the cumulative prevalence of all persons infected in the population.

\section{Statistical analysis}

Data analyses were done using Graph Pad Prism statistical software $\odot$ (Version 5). The microfilaria prevalence was expressed as a percentage (number of persons positive for microfilaria divided by number examined $\times 100$ ). Chi-square analysis, ANOVA and Welch's unequal variances $t$ test were used to test for differences in microfilaria and nodule prevalence within and between the study communities. A two-tailed $p$-value lower than 0.05 was considered statistically significant.

\section{Results}

\section{Demographic data of study communities Gender}

In total 114 participants were included in the study from all the three study communities, of which $50.88 \%$ ( $n=$ 58 ) were females and $49.12 \%$ were males $(n=56)$.

\section{Age distribution of study participants}

The age distributions of study participants in all three study communities are presented in Table 1 . In the Senya community, the study showed that the age group 51-61 years had the highest frequency of 14 representing about $34.15 \%$ of the total population sampled in the community (Table 1). The lowest frequency of participants (4) was recorded in the 29-39 years category.

In the Kokompe community, the study found out that the greater majority of the participants sampled were in the 62-95 year range with a frequency of 13 (representing $37.14 \%$ of the sampled population). The $18-28$ year
Table 1 Frequency Distribution of Ages of Study Participants in Senya, Kokompe and Tanfiano Communities

\begin{tabular}{lllll}
\hline $\begin{array}{lllll}\text { Age } \\
\text { Group }\end{array}$ & \multicolumn{2}{l}{ Frequency (percent) } & \\
\cline { 2 - 4 } & Senya & Kokompe & Tanfiano & Total \\
\hline $18-28$ & $8(19.5 \%)$ & $1(2.9 \%)$ & $7(18.4 \%)$ & $16(14.0 \%)$ \\
$29-39$ & $4(9.8 \%)$ & $4(11.4 \%)$ & $8(21.1 \%)$ & $16(14.0 \%)$ \\
$40-50$ & $9(22.0)$ & $11(31.4 \%)$ & $8(21.1 \%)$ & $28(24.6 \%)$ \\
$51-61$ & $14(34.2 \%)$ & $6(17.1 \%)$ & $7(18.4 \%)$ & $27(23.7 \%)$ \\
$62-95$ & $6(14.6 \%)$ & $13(37.1 \%)$ & $8(21.1 \%)$ & $27(23.7 \%)$ \\
\hline
\end{tabular}

category recorded only one (1) person representing about $2.86 \%$ of the sampled population (Table 1 ).

In the Tanfiano community, the study showed that the frequency of participants in the various age categories were similar (Table 1 ). The age categories $29-39,40-50$ and 62-95 all had frequencies of 8 each representing $21.05 \%$ of the total patients sampled in the community.

\section{Parasitological indices of onchocerciasis}

The parasitological indices of onchocerciasis within the study population $(n=114)$ is summarized in Table 2 . It is noteworthy that every community in this study had MDA ongoing for more than 10 years. For the entire study population, there were 7 people $(6 \%)$ who were carriers of microfilaria. Tanfiano had the highest prevalence of microfilaria carriers $(13.2 \% ; n=5)$, followed by Kokompe $(2.9 \% ; \mathrm{n}=1)$ and Senya $(2.43 \% ; \mathrm{n}=1)$, but the difference was not statistically significant $(p=0.2717$, one-way ANOVA).

The association between nodule prevalence and microfilaria prevalence was variable. The Tanfiano community recorded the highest microfilaria prevalence among the study communities. The microfilaria prevalence in Tanfiano was $13.2 \%$, which was more than 2 times higher than the prevalence of onchocercal nodules (5.3\%), though the difference was not statistically significant $(p=0.8553$, Welch's unequal variances $t$-test). In Senya, the prevalence of onchocercal nodules was $4.9 \%$, about 2 times higher than the microfilaria prevalence which was $2.4 \%$, however, the difference was not statistically significant ( $p=0.2546$, Welch's unequal variances $t$-test). In Kokompe however, the prevalence

Table 2 The Prevalence of Nodules and Microfilaria ( $\mathrm{mf}$ ) of the Study Population

\begin{tabular}{lllll}
\hline $\begin{array}{l}\text { Study } \\
\text { Community }\end{array}$ & $\begin{array}{l}\text { Rounds of } \\
\text { ivermectin }\end{array}$ & $\begin{array}{l}\text { No. of } \\
\text { cases } \\
\text { examined }\end{array}$ & $\begin{array}{l}\text { Prevalence of } \\
\text { nodule careers } \\
\text { N; }(\%)\end{array}$ & $\begin{array}{l}\text { Prevalence of } \\
\text { Mf carriers } \\
\text { N;(\%) }\end{array}$ \\
\hline Tanfiano & 20 & 38 & $2(5.3 \%)$ & $5(13.2 \%)$ \\
Senya & 20 & 41 & $2(4.9 \%)$ & $1(2.4 \%)$ \\
Kokompe & 18 & 35 & $5(14.3 \%)$ & $1(2.9 \%)$ \\
Total & - & 114 & $9(7.89 \%)$ & $7(6.14 \%)$ \\
\hline
\end{tabular}


of nodules (14.3\%) was about 5 times higher than the microfilaria prevalence (2.9\%) albeit the difference was just not statistically significant $(p=0.0580$, Welch's unequal variances $t$-test).

\section{Gender distribution of nodules and microfilaria in the study communities}

The prevalence of nodules and microfilaridermia among females and males in the study communities is presented in Table 3. Females in the study communities had a higher prevalence of microfilaria carriers (8.6\%) relative to that of the males (5.4\%), though the difference was not statistically significant $(p=0.7526$, Chi Square test). The prevalence of nodules was slightly higher in females (8.6\%) compared to males (7.1\%), however, again the difference was not statistically significant $(p=0.7699$, Chi Square test).

\section{Clinical manifestations of onchocerciasis in the study communities}

The summary of findings on onchocerciasis-associated clinical manifestations are presented in Table 4. The most frequent clinical manifestation observed in this study was dermatitis. This was followed by ocular onchocerciasis (visual impairment and blindness). The percentage of individuals with dermal and ocular onchocerciasis in the 3 study areas were $33.3 \%$ (38/114) and $12.3 \%$ (14/114) respectively.

The relationship between dermatitis and microfilaredermia is presented in Table 5. A large number of nonmicrofilaridermic people also had dermatitis as 25 people who were microfilaria negative reported that they experienced dermatitis. The number of people who were microfilaria positive and had episodes of dermatitis was almost the same as those who were microfilaria positive but did not experience any dermatitis.

\section{Discussion}

This study is the first published work on the prevalence of onchocerciasis and its associated clinical manifestations in the Nkoranza North District of the Bono East Region. Thus, the findings are important to help direct the onchocerciasis control programme of the district as well as nearby districts with similar characteristics.

The study demonstrated that the microfilaria level for all 3 communities was below $20 \%$. This means that the

Table 3 Gender Distribution of Nodules and Microfilaria Prevalence in the Study Communities

\begin{tabular}{llll}
\hline Gender & $\begin{array}{l}\text { No. of cases } \\
\text { examined }\end{array}$ & $\begin{array}{l}\text { Prevalence of Mf } \\
\text { Carriers N; (\%) }\end{array}$ & $\begin{array}{l}\text { Prevalence of nodule } \\
\text { carriers (\%) }\end{array}$ \\
\hline Females & 58 & $5(8.6 \%)$ & $5(8.6 \%)$ \\
Males & 56 & $3(5.4 \%)$ & $4(7.1 \%)$ \\
Total & 114 & $8(7 \%)$ & $9(7.9 \%)$ \\
\hline
\end{tabular}

study areas are hypoendemic for onchocerciasis based on the criteria used by the Onchocerciasis Elimination Programme for the Americas (OEPA), where nodule and microfilarial prevalence of $\leq 20 \%, 21-59 \%$ and $\geq 60 \%$ are defined as hypoendemic, mesoendermic and hyperendemic respectively [27]. This finding is interesting because the district of the study area was previously classified as mesoendemic for onchocerciasis by the Ghana Health Service [28]. Consequently, the present observations suggest a general downward change in prevalence of the disease. This supports the frequently reported effectiveness of ivermectin as a microfilaricide. However, the community of Tanfiano which had received over 20 rounds of ivermectin had a higher (though statistically not significant) microfilaria prevalence than the other two communities. This finding could be due to the fact that the community frequently received quite a number of migrants from areas such as Kintampo, Atebubu, Prang and other surrounding communities [29] which are known to be hyper or mesoendemic for onchocerciasis.

In this study, the prevalence of both microfilaria and nodules were higher in females than in males. Gender related nodule prevalence has been reported in other studies and it has been attributed to the differences in degree and frequency of duration of how males and females participants are exposed to the bites of infective Simulium vectors, with female's participants being more exposed [30, 31]. Their increased exposure can be due to certain roles and activities such as washing by river banks and fetching water for drinking and domestic purposes [30,31]. However, some other studies have found contrasting results as the prevalence of onchocerciasis in males in those areas were higher than the prevalence in females. The differential exposure to bites of blackfly fly vectors has been shown to be dependent on the behaviour, occupation and clothing of different gender which vary from one culture to the next [32].

The study also investigated the prevalence of certain clinical manifestations which are often associated with river blindness. It was found that the most frequently encountered clinical symptom in the study communities was dermatitis, followed by visual impairment, nodules and blindness. Visual damage is considered to be the most serious clinical feature of onchocerciasis, which may affect all tissues of the eye [4]. Ocular lesions are usually seen only in those with moderate or heavy microfilarial loads [33]. Although onchocerciasis is commonly known as river blindness, the ocular manifestations of the disease actually only occur in about $5 \%$ of those affected [4]. However for this study, the prevalence of ocular manifestations is $12 \%$ which is relatively higher than the normal prevalence of ocular onchocerciasis frequently reported [4], though other authors have reported higher estimates in other countries [34]. 
Table 4 Clinical Manifestations of Onchocerciasis in the Three Study Communities of the Nkoranza North District of Ghana

\begin{tabular}{llllll}
\hline Study Community & No. of cases examined & No. of people with Nodules (N; \%) & Dermatitis (N;\%) & Visual impairment (N;\%) & Blindness (N;\%) \\
\hline Tanfiano & 38 & $2(5.3 \%)$ & $6(15.8 \%)$ & $4(10.5 \%)$ & $0(0.0 \%)$ \\
Senya & 41 & $2(4.9 \%)$ & $12(29.3 \%)$ & $3(7.3 \%)$ & $2(4.9 \%)$ \\
Kokompe & 35 & $5(14.3)$ & $11(31.4 \%)$ & $2(5.7 \%)$ & $3(8.5 \%)$ \\
Total & 114 & $9(7.89 \%)$ & $29(25.44 \%)$ & $9(7.89 \%)$ & $5(4.4 \%)$ \\
\hline
\end{tabular}

Another interesting finding from this study was the fact that a large number of amicrofilaridermic people also had dermatitis. This might be possible because ivermectin is a known effective microfilaricide, as it is able to clear skin microfilaria and in some places has been proven to halt transmission of onchocerciasis $[15,18,19]$. Thus, people infected with onchocerciasis who take ivermectin during MDAs might have the microfilaria cleared from their skin, though some of the chronic clinical manifestations of onchocerciasis might persist. However, despite the strong microfilaricidal and embryostatic effects of ivermectin, several investigators have demonstrated that it has negligible or no macrofilaricidal effects [35-37]. This has necessitated the search for new or repurposed antifilarial drugs which have adulticidal effects on $O$. volvulus worms [38]. In this respect, the use of doxycycline which has been proven to have adulticidal effects on Wolbachia can be considered as doxycycline results in amelioration of clinical manifestations of the disease [38]. There is however the need for more research in this regard as the usage of doxycycline in MDA programmes has some logistical challenges [38]. Some of these challenges include contraindications in children below 8 and pregnant women as well as concerns that if doxycycline is used on a large scale such as in MDA programmes, resistance to the antibiotic might develop [39]. Meanwhile, doxycycline remains one of the major "weapons" for the treatment of a variety of atypical infections $[39,40]$.

\section{Conclusions}

The results from this study demonstrated that onchocerciasis remains a health problem in the study area despite several years of ivermectin administration. Though the drug seems to be effective in reducing microfilaria prevalence in the communities, the persistence of the disease

Table 5 The Relationship between Dermatitis and Microfilaridermia among the Study Participants

\begin{tabular}{llll}
\hline Data analyzed & MF positive & MF Negative & Total \\
\hline Dermatitis & 4 & 25 & 29 \\
No Dermatitis & 3 & 82 & 85 \\
Total & 7 & 107 & 114 \\
\hline
\end{tabular}

provides some cause for concern. Constant surveillance of the disease is thus warranted in order to help review the frequency of MDA with ivermectin in the district. There is also an increasing urgency for research and development towards new antifilarial drugs which have macrofilaricidal effects to complement ivermectin.

\section{Abbreviations}

DALYs: Disability adjusted life years; MDA: Mass drug administration; OEPA: Onchocerciasis Elimination Programme for the Americas; SOR: Suboptimal response; WHO: World Health Organization

\section{Acknowledgements}

We are grateful to all staff of the Department of Community Medicine and Health of the Anglican University College of Technology, Nkoranza, Ghana for the various contributions that resulted in this publication. We also appreciate the goodwill and support of the staff of the Department of Basic and Applied Biology of the University of Energy and Natural Resources, Sunyani, Ghana. The study also appreciates the kindness of the Nkoranza North Health Directorate, the chiefs, the health volunteers and people of Tanfiano, Senya and Kokompe for their diverse support which resulted in the completion of this study.

\section{Funding}

Not applicable.

\section{Availability of data and materials}

The datasets used and/or analyzed during the current study are available from the corresponding author on reasonable request.

\section{Authors' contributions}

$\mathrm{KBO}, \mathrm{HDFHS}$, SFG conceived and designed the study and drafted the manuscript. EA and DO-O reviewed the study design and methodology and critically reviewed the manuscript. RA-NJ, DA, BC, LA, PA, CBA, A-RN collected data in the field, managed, secured and transferred all collected data and aided in data analysis. All authors have approved the final manuscript.

Ethics approval and consent to participate

All participants gave a signed informed consent to participate. This study received the ethical approval of the Kintampo Health Research Centre, Kintampo, Brong Ahafo Region, Ghana.

Consent for publication

Not applicable.

Competing interests

The authors declare that they have no competing interests.

\section{Publisher's Note}

Springer Nature remains neutral with regard to jurisdictional claims in published maps and institutional affiliations.

\footnotetext{
Author details

${ }^{1}$ Department of Basic and Applied Biology, School of Science, University of Energy and Natural Resources, Sunyani, Ghana. ${ }^{2}$ Amsterdam University Medical Centres, Academic Medical Centre, University of Amsterdam, Department of Medical Microbiology, Experimental Parasitology Unit, Amsterdam, The Netherlands. ${ }^{3}$ Department of Civil Engineering, Kwame
} 
Nkrumah University of Science and Technology, Kumasi, Ghana. ${ }^{4}$ Office of the Vice Chancellor, Catholic University College of Ghana, Sunyani, Ghana. ${ }^{5}$ Department of Community Medicine and Health, Anglican University College of Technology, Nkoranza, Ghana.

\section{Received: 1 February 2019 Accepted: 9 May 2019} Published online: 17 May 2019

\section{References}

1. WHO, "Onchocerciasis," 2018. [Online]. Available: https://www.who.int/newsroom/fact-sheets/detail/onchocerciasis. [Accessed 27 Jan 2019].

2. Osei-Atweneboana MY, Eng JK, Boakye DA, Gyapong JO, Prichard RK. Prevalence and intensity of Onchocerca volvulus infection and efficacy of ivermectin in endemic communities in Ghana: a two-phase epidemiological study, 2021-2029. 2007.

3. Remme JHF, et al. Tropical Diseases Targeted for Elimination: Chagas Disease, Lymphatic Filariasis, Onchocerciasis, and Leprosy. The International Bank for Reconstruction and Development / The World Bank; 2006.

4. Gillespie SH, Pearson RD. Principles and practice of clinical parasitology: Wiley; 2001.

5. Okulicz JF, Elston DM and Schwartz RA. "Dermatologic manifestations of onchocerciasis (river blindness): background, pathophysiology, etiology," 2018. [Online]. Available: https://emedicine.medscape.com/article/1109409overview\#section clinical. Accessed 26 Jan 2019.

6. Thylefors B. The Mectizan donation program (MDP). Ann Trop Med Parasitol. 2008;102(sup1):39-44.

7. Awadzi $K$, et al. Thirty-month follow-up of sub-optimal responders to multiple treatments with ivermectin, in two onchocerciasis-endemic foci in Ghana. Ann Trop Med Parasitol. 2004;98(4):359-70.

8. Osei-Atweneboana MY, Boakye DA, Awadzi K, Gyapong JO, Prichard RK. Genotypic analysis of $\beta$-tubulin in Onchocerca volvulus from communities and individuals showing poor parasitological response to ivermectin treatment. Int J Parasitol Drugs Drug Resist. 2012;2:20-8.

9. Osei-Atweneboana MY, Awadzi K, Attah SK, Boakye DA, Gyapong JO, Prichard RK. Phenotypic evidence of emerging ivermectin resistance in onchocerca volvulus. PLoS Negl Trop Dis. 2011;5(3):e998.

10. Awadzi $\mathrm{K}$, et al. An investigation of persistent microfilaridermias despite multiple treatments with ivermectin, in two onchocerciasis-endemic foci in Ghana. Ann Trop Med Parasitol. 2004:98(3):231-49.

11. Doyle SR, et al. Genome-wide analysis of ivermectin response by Onchocerca volvulus reveals that genetic drift and soft selective sweeps contribute to loss of drug sensitivity. PLoS Negl Trop Dis. 2017;11(7): e0005816. https://doi.org/10.1371/journal.pntd.0005816.

12. Frempong KK, et al. Does increasing treatment frequency address suboptimal responses to Ivermectin for the control and elimination of river blindness? Clin Infect Dis. 2016;62(11):1338-47.

13. Nana-Djeunga $\mathrm{HC}$, et al. Reproductive status of Onchocerca volvulus after ivermectin treatment in an ivermectin-naïve and a frequently treated population from Cameroon. PLoS Negl Trop Dis. 2014;8(4):e2824.

14. Pion SDS, et al. Dynamics of Onchocerca volvulus microfilarial densities after Ivermectin treatment in an Ivermectin-naïve and a multiply treated population from Cameroon. PLoS Negl Trop Dis. 2013;7(2):e2084.

15. Ardelli BF, Prichard RK. Identification of variant ABC-transporter genes among Onchocerca volvulus collected from ivermectin-treated and untreated patients in Ghana, West Africa. Ann Trop Med Parasitol. 2004; 98(4):371-84.

16. Ardelli BF, Guerriero SB, Prichard RK. Ivermectin imposes selection pressure on P-glycoprotein from Onchocerca volvulus: linkage disequilibrium and genotype diversity. Parasitology. 2006;132(Pt 3):375-86.

17. Ardelli BF, Guerriero SB, Prichard RK. Characterization of a half-size ATPbinding cassette transporter gene which may be a useful marker for ivermectin selection in Onchocerca volvulus. Mol Biochem Parasitol. 2006; 145(1):94-100

18. Ardelli BF, Guerriero SB, Prichard RK. Genomic organization and effects of ivermectin selection on Onchocerca volvulus P-glycoprotein. Mol Biochem Parasitol. 2005;143(1):58-66.

19. Ardelli and Prichard. Reduced genetic variation of an Onchocerca volvulus $A B C$ transporter gene following treatment with ivermectin. Trans R Soc Trop Med Hyg. 2007;101(12):1223-32.
20. Bourguinat C, et al. P-glycoprotein-like protein, a possible genetic marker for ivermectin resistance selection in Onchocerca volvulus. Mol Biochem Parasitol. 2008;158(2):101-11.

21. Ghana Statistical Service. 2010 Population and housing census: District analytical report for Nkoranza district, 2010. [Online]. Available: http://www2. statsghana.gov.gh/docfiles/2010_District_Report/Brong\%20Ahafo/ NKORANZA\%20North.pdf. Accessed 27 Jan 2019.

22. Stanton CK, et al. Impact on postpartum hemorrhage of prophylactic administration of oxytocin $10 \mathrm{IU}$ via UnijectTM by peripheral health care providers at home births: design of a community-based cluster-randomized trial. BMC Pregnancy Childbirth. 2012;12(1):42.

23. WHO. Guidelines for revising ivermectin treatment. 2018. Available: https:// www.who.int/apoc/ATS_Report_Annex1_APOC_Guidelines_for_revising_ IVM_Tx_boundaries.pdf. Accessed 27 Jan 2019.

24. Albiez EJ. Studies on nodules and adult Onchocerca volvulus during a nodulectomy trial in hyperendemic villages in Liberia and upper Volta. I. Palpable and impalpable onchocercomata. Tropenmed Parasitol. 1983; 34(1):54-60.

25. Sue S. Test distance vision using a Snellen chart. Commun Eye Heal. 2007; 20(63):52.

26. Noormahomed EV, Akrami K, Mascaró-Lazcano C. Onchocerciasis, an undiagnosed disease in Mozambique: identifying research opportunities. Parasit Vectors. 2016:1-8.

27. Sauerbrey M. The onchocerciasis elimination program for the Americas (OEPA). Ann Trop Med Parasitol. 2008;102(sup1):25-9.

28. Taylor MJ, et al. Onchocerciasis control: vision for the future from a Ghanian perspective. Parasit Vectors. 2009;2(1):7.

29. Adu-Boahen K. Nkoransa, C1700-1900: A study of its formation and relation with its neighbours. A thesis submitted to the University of Cape Coast, Ghana, 1997. Available: https://erl.ucc.edu.gh/jspui/bitstream/123456789/ 1450/1/ADU-BOAHEH\%201997.pdf. Accessed 15 May 2019.

30. Anosike JC, Celestine O, Onwuliri E, Onwuliri VA. The prevalence, intensity and clinical manifestations of Onchocerca volvulus infection in Toro local government area of Bauchi state, Nigeria. Int J Hyg Environ Health. 2001; 203(5-6):459-64.

31. Opara KN, Usip LP, Akpabio EE. Transmission dynamics of Simulium damnosum in rural communities of Akwa Ibom State, Nigeria. 2008. p. 22530 .

32. Filipe JAN, et al. Human infection patterns and heterogeneous exposure in river blindness. Proc Natl Acad Sci U S A. 2005;102(42):15265-70.

33. Anderson J, Fuglsang H, Hamilton PJ, de Marshall TF. Studies on onchocerciasis in the United Cameroon Republic. II. Comparison of onchocerciasis in rain-forest and Sudan-savanna. Trans R Soc Trop Med Hyg. 1974:68(3):209-22

34. Umeh RE, et al. Prevalence and distribution of ocular onchocerciasis in three ecological zones in Nigeria. Afr J Med Med Sci. 2010;39(4):267-75.

35. Dreyer G, Amaral F, Norões J, Medeiros Z, Addiss D. A new tool to assess the adulticidal efficacy in vivo of antifilarial drugs for bancroftian filariasis. Trans R Soc Trop Med Hyg. 1995;89(2):225-6.

36. Plaisier AP, Cao WC, van Oortmarssen GJ, Habbema JD. Efficacy of ivermectin in the treatment of Wuchereria bancrofti infection: a modelbased analysis of trial results. Parasitology. 1999;119(Pt 4):385-94.

37. Richard-Lenoble D, Chandenier J, Gaxotte P. Ivermectin and filariasis. Fundam Clin Pharmacol. 2003;17(2):199-203.

38. Hoerauf A. Filariasis: new drugs and new opportunities for lymphatic filariasis and onchocerciasis. 2008

39. Leggat PA. Clinical medicine: therapeutics safety and efficacy of doxycycline. 2009. p. 1069-72.

40. Holmes NE, Charles PGP. Safety and efficacy review of doxycycline. Clin Med Ther. 2009;1:CMTS2035. 